\title{
Comparing optimized renormalization schemes for QCD observables
}

\author{
M. Akrami ${ }^{\dagger}$ and A. Mirjalili॰* \\ Physics Department, Yazd University, P.O. Box 89195-741, Yazd, Iran
}

(Received 1 October 2019; accepted 14 January 2020; published 10 February 2020)

\begin{abstract}
In this article, based on the approach of McKeon et al., we show that the renormalization group equation related to the radiative mass scale $\mu$ operates as a summation over QCD perturbative terms. Employing the full QCD $\beta$ function within this summation, all logarithmic corrections can be presented as log-independent contributions. In another step of this approach, the renormalization scheme dependence for QCD observables characterized by Stevenson can be examined by specifying the renormalization scheme, in which the $\beta$ function beyond two-loop order is utilized. In this regard, there are two choices of renormalization scheme. In the first scheme, the QCD observable involves two powers of the running coupling constant, such that the perturbative series contains just two terms. In the second scheme, the perturbative series expansion is written as an infinite series in terms of the two-loop running coupling, which can be represented by the Lambert $W$ function. In both cases, the QCD observable involves parameters that are renormalization scheme invariant and the coupling constant, which is independent of the renormalization scale. We then consider another approach, which is called complete renormalization group improvement (CORGI). In this approach, by using the self-consistency principle it is possible to reconstruct the conventional perturbative series in terms of scheme-invariant quantities and the coupling constant as a function of the Lambert $W$ function. It should be noted that while in the renormalization group summation method of McKeon et al. the scheme dependence of observables is investigated separately from their scale dependence, in the CORGI approach, through the principle of self-consistency, both scale and scheme parameters are utilized. Then, we numerically examine these two approaches, considering two QCD observables. The first is the $R_{e^{+} e^{-}}$ratio, investigated at three different colliding energies, and the second is the Higgs decay width to a gluon pair. We compare our results for $R_{e^{+} e^{-}}$with the available experimental data. The results based on the McKeon et al. approach are in better agreement with the experimental data.
\end{abstract}

DOI: 10.1103/PhysRevD.101.034007

\section{INTRODUCTION}

There are different approaches to optimizing QCD observables. One of them is called the principle of maximum conformality (PMC), which was developed by Brodsky and collaborators [1-5]. In this approach the perturbative series is divided into a conformal and a nonconformal part. The conformal part involves terms that are independent of the employed scheme. The nonconformal part is absorbed into the coupling constant by choosing proper scales. During the absorbtion, the renormalization scales are fixed and determined, and the final result is such

\footnotetext{
* Corresponding author. A.Mirjalili@yazd.ac.ir

†Akrami@stu.yazd.ac.ir

Published by the American Physical Society under the terms of the Creative Commons Attribution 4.0 International license. Further distribution of this work must maintain attribution to the author(s) and the published article's title, journal citation, and DOI. Funded by SCOAP ${ }^{3}$.
}

that the conformal series is independent of the scheme and scales used. The division of the series can be done using an auxiliary scheme called the $R_{\delta}$ scheme [2]. Some research activities related to the PMC approach can be found in Refs. [6,7].

Another approach to optimizing QCD observables is called complete renormalization group improvement (CORGI) [8]. In this approach, using the self-consistency principle, it is possible to write each expansion coefficient at high order in terms of coefficients of lower orders and invariant scheme-independent terms, which are unknown at that high order. If one resums the contribution of each expansion term at lower orders, it can be shown that the result is scale and scheme independent. This provides an opportunity to reconstruct the initial perturbative series in terms of scale- and scheme-independent terms and the coupling constant which is determined at physical energy scale, and consequently the scale ambiguity is removed.

There might be some connections between the PMC and CORGI approaches, which have been discussed in Ref. [9]. The most important one is that the predictable terms in the 
CORGI approach can be assigned to the nonconformal part since the predictable terms are scale and scheme dependent, while the unpredictable terms can be related to the conformal part of the PMC approach, which is scheme independent. For more details, see Ref. [9].

In a third approach developed by McKeon and his collaborators-which we call the "McKeon et al. approach"-it can be shown that upon resummation of all perturbative series terms to all orders, using the full QCD $\beta$ function, the result is scale independent and only depends on the physical energy scale $Q$, without any ambiguity. Using the scheme dependence of the coupling constant, it is shown that the perturbative coefficients, at a specified high order, can be written in terms of coefficients at lower orders up to the first order coefficient [10-14]. This situation is like that in the CORGI approach, but with one little difference. In the CORGI approach the perturbative coefficients at some specified high order are obtained in terms of coefficients at lower orders based on the selfconsistency principle. In this regard, it is necessary to obtain the derivative of the perturbative coefficients with respect to not only the scheme parameters, but also the renormalization scale which is cast in terms of the variable $\tau$ (see Ref. [15]). Therefore, in comparison with the McKeon et al. approach, the result of the CORGI approach for the perturbative coefficients at a specified high order not only contains the coefficients at lower orders, but also involves some extra terms. AS mentioned above, this occurs because the separation between the scale and scheme dependence does not happen during the optimization procedure in the CORGI approach, but it does in the McKeon et al. approach. In this paper we discuss in more detail the differences and similarities between the CORGI and McKeon et al. approaches and investigate which one is the preferred method. In particular, we examine these procedures by considering two QCD observables: the ratio of electron-positron annihilation to hadrons, and the Higgs decay width to a gluon pair. But first we need to review the basic concepts of theses two approaches, which we do in the following sections.

This paper is organized as follows. In Sec. II we review the basic concepts of the McKeon et al. approach. This section contains two parts. In Sec. II A we describe the renormalization group (RG) summation, and in Sec. II B we describe the scheme used to characterize the QCD observables. In Sec. III, we examine the McKeon et al. and CORGI approaches for some QCD observables. In Secs. III A and III B we investigate the ratio $R_{e^{+}\left(e^{-}\right)}$ratio in the McKeon et al. and CORGI approach, respectively. In Sec. IV, after describing how to perform the RG resummation, we use the McKeon et al. approach to study the Higgs decay width to a gluon pair. The scheme dependence for this observable is determined in Sec. IV $\mathrm{A}$, and a numerical investigation of this observable using the McKeon et al. approach is presented in Sec. IV B. In
Sec. V we present a numerical investigation of this observable using the CORGI approach. Finally, we give our conclusion in Sec. VI.

\section{AN OVERVIEW OF THE MCKEON ET AL. APPROACH}

Here we give a brief description of the renormalization group summation method developed by McKeon et al. For clarity of presentation, most of the relations in this and the following sections are adapted from Refs. [10,11]. As an example of how the renormalization group summation is done, we examine the QCD observable for $e^{+} e^{-}$annihilation. In the following, we show that by determining a recurrence relation between perturbative terms, the result of the summation of all perturbative terms is independent of the renormalization scale as an unphysical parameter.

The cross section of $e^{+} e^{-}$annihilation into hadrons, after normalizing it to $\mu^{+} \mu^{-}$pair production, can be written as

$$
R_{e^{+} e^{-}}=\left(3 \sum_{i} q_{i}^{2}\right)[1+R] .
$$

The expansion of $R$ in terms of the coupling constant $a$ has the following form:

$$
R=\sum_{n=0}^{\infty} r_{n} a^{n+1}, \quad r_{0}=1 .
$$

The coefficient of $r_{n}$, arising from the contributions of Feynman diagrams to the observable $R$, can be represented by [10]

$$
r_{n}=\sum_{m=0}^{n} T_{n m} L^{m} .
$$

In this equation $T_{00}=1$ and $L=b \ln \left(\frac{\mu}{Q}\right)$, where $\mu$ is the renormalization scale and $Q$ is the physical center-of-mass energy in the $e^{+} e^{-}$collision.

Since $R$ is independent of the renormalization scale $\mu$, the renormalization group equation (RGE) implies that

$$
\mu \frac{d}{d \mu} R=\left(\frac{\partial}{\partial \mu}+\beta(a) \frac{\partial}{\partial a}\right) R=0 .
$$

The $\mu$ dependence of the coupling is governed by the QCD $\beta$-function equation [16]:

$\beta(a)=\mu \frac{\partial a}{\partial \mu}=-b a^{2}\left(1+c a+c_{2} a^{2}+c_{3} a^{3}+\cdots\right)$.

Here $b=\left(33-2 N_{f}\right) / 6$ and $c=\left(153-19 N_{f}\right) / 12 b$ are renormalization scheme (RS) invariant, where $N_{f}$ denotes the number of active quark flavors. The higher coefficients $c_{2}, c_{3}, \ldots$ serve to label the RS dependence [17]. 
To indicate that the coefficients $b$ and $c$ in Eq. (5) are scheme independent, one can consider two different couplings $a$ and $a^{*}$ at two different schemes, such that [11]

$$
a^{*}=a+x_{2} a^{2}+x_{3} a^{3}+\cdots,
$$

and therefore

$$
\beta^{*}\left(a^{*}\right)=\mu \frac{\partial a^{*}}{\partial \mu}=\beta(a) \frac{\partial a^{*}}{\partial a} .
$$

Then, one can show that

$$
\begin{aligned}
\beta^{*}\left(a^{*}\right)= & -b^{*} a^{* 2}\left[1+c^{*} a^{*}+c_{2}^{*} a^{* 2}+\cdots\right] \\
= & {\left[1+2 x_{2} a+3 x_{3} a^{2}+\cdots\right]\left(-b a^{2}\right) } \\
& \times\left[1+c a+c_{2} a^{2}+\cdots\right] .
\end{aligned}
$$

If we substitute $a^{*}$ from Eq. (6) into the first row of Eq. (8) and compare the result with the second row of this equation, we arrive at

$$
\begin{aligned}
b & =b^{*}, \\
c & =c^{*}, \\
c_{2} & =c_{2}^{*}+c x_{2}+x_{2}^{2}-x_{3},
\end{aligned}
$$

We now deal with the details of the McKeon et al. approach.

\section{A. Renormalization group summation}

Using the McKeon et al. approach, it can be shown that by a full resummation of the QCD perturbative series the unphysical parameter $\mu$ can be removed and the final result for any QCD observable is independent of the unphysical parameter, as is expected. One of the features of the McKeon et al. approach compared to the CORGI approach is that it does not require the use of the self-consistency principle, and in order to construct the McKeon et al. approach, it only needs to utilize RGE by considering the QCD $\beta$ function. In the CORGI approach, one needs to employ the self-consistency principle for not only the scheme parameters but also the scale parameter $\mu$. Therefore, the scale and scheme parameters are used simultaneously during the optimization process in the CORGI approach, while in the McKeon et al. approach the renormalization scale parameter and scheme parameters are used separately.

Now we briefly review the McKeon et al. approach to show how the unphysical parameters are removed in perturbative series for any QCD observable, using just the RGE of QCD $\beta$ function. For this purpose, by substituting Eq. (3) into Eq. (2) we obtain [10]

$$
R=R_{\text {pert }}=\sum_{n=0}^{\infty} r_{n} a^{n+1}=\sum_{n=0}^{\infty} \sum_{m=0}^{n} T_{n, m} L^{m} a^{n+1} .
$$

To satisfy the condition $r_{0}=1 \mathrm{in} \mathrm{Eq.} \mathrm{(2),} \mathrm{it} \mathrm{is} \mathrm{required} \mathrm{that}$ $T_{0,0}=1$. From Eq. (10), the following expression should be assigned to $r_{n}$ :

$$
r_{n}=\sum_{m} T_{n, m} L^{m}
$$

If one sets $m=0$, then

$$
r_{n}=T_{n} \Rightarrow R=\sum_{n=0}^{\infty} T_{n} a^{n+1},
$$

which is similar to Eq. (2).

Now we introduce a new grouping, denoted by $A_{n}[10]$ :

$$
A_{n}=\sum_{m=0}^{\infty} T_{n+m, n} a^{n+m+1}
$$

This makes it possible to sum the contribution to $R$ by considering the RGE. Therefore, using Eq. (13) in the above, the $R$ in Eq. (10) is expressed as

$$
R=R_{A}=\sum_{n=0}^{\infty} A_{n}(a) L^{n}
$$

By substituting Eq. (14) into the renormalization group equation (4), it can be shown that

$$
\sum_{n=0}^{\infty}\left(b n A_{n}(a) L^{n-1}+\beta(a) A_{n}^{\prime}(a) L^{n}\right)=0 .
$$

Using this equation and rearranging the order of sums, $A_{n}$ can be written as

$$
A_{n}(a)=-\frac{\beta(a)}{n b} \frac{d}{d a} A_{n-1}(a) .
$$

Considering the QCD $\beta$ function (5), we find that

$$
A_{n}\left(a\left(\ln \frac{\mu}{\Lambda}\right)\right)=-\frac{1}{n} \frac{d}{d \ln \left(\frac{\mu}{\Lambda}\right)} A_{n-1}\left(a\left(\ln \frac{\mu}{\Lambda}\right)\right),
$$

where $\Lambda$ is related to be boundary condition in Eq. (5) such that 


$$
\ln \left(\frac{\mu}{\Lambda}\right)=\int_{0}^{a} \frac{d x}{\beta(x)}+\int_{0}^{\infty} \frac{d x}{b x^{2}(1+c x)}
$$

Defining $\eta=\ln \frac{\mu}{\Lambda}$ and using the recurrence relation (17), one obtains

$$
A_{n}(a(\eta))=\frac{-1}{b n} \frac{d}{d \eta} A_{n-1}(a(\eta))=\frac{1}{n !}\left(-\frac{1}{b} \frac{d}{d \eta}\right)^{n} A_{0}(a(\eta)) .
$$

Substituting Eq. (19) into Eq. (14) gives

$$
R_{A}=\sum_{n=0}^{\infty} \frac{1}{n !}\left(-\frac{L}{b}\right)^{n} \frac{d^{n}}{d \eta^{n}} A_{0}(a(\eta))=A_{0}\left(a\left(\eta-\frac{L}{b}\right)\right)
$$

To prove the above relation, it is easy to show that

$$
\begin{aligned}
R(A) & =\sum_{n=0}^{\infty} \frac{1}{n !}\left(-\frac{L}{b}\right)^{n} \frac{d^{n}}{d \eta^{n}} A_{0}(a(\eta)) \\
& =\exp \left(\left(-\frac{L}{b}\right) \frac{d}{d \eta}\right) A_{0}(a(\eta))
\end{aligned}
$$

On the other hand, using the Taylor expansion for the righthand side of Eq. (20), one can write

$$
\begin{aligned}
A_{0}\left(a\left(\ln \frac{\mu}{\Lambda}-L\right)\right)= & A_{0}\left(a\left(\ln \frac{\mu}{\Lambda}\right)\right)-L A_{0}^{\prime}\left(a\left(\ln \frac{\mu}{\Lambda}\right)\right) \\
& +\frac{L^{2}}{2 !} A_{0}^{\prime \prime}\left(a\left(\ln \frac{\mu}{\Lambda}\right)\right)+\cdots,
\end{aligned}
$$

which is equivalent to the right-hand side of Eq. (21), considering the expansion of the exponential term. Consequently, based on the definitions of the parameters $L$ and $\eta$, one can see that [11]

$$
R_{A}=A_{0}\left(a\left(\ln \frac{Q}{\Lambda}\right)\right)
$$

This equation shows that all of the dependence of $R$ on $\mu$ scale has been canceled. This is a pleasant result since $\mu$ is an unphysical parameter and it is expected to be removed by doing a full resummation of the QCD perturbative series.

\section{B. Renormalization scheme dependence}

In the previous section we showed that the final result for a perturbative series of any QCD observable is independent of the renormalization scale $\mu$ as an unphysical parameter. Here we briefly review the McKeon et al. approach to show why the perturbative series of a QCD observable is scheme independent, and therefore that the final result for the QCD perturbative series is reliable and independent of the scale and scheme parameters.

Let us to start by recalling that $b$ and $c$, the first two parameters in the QCD $\beta$ function, are independent of the renormalization scheme, while the expansion parameters $c_{i}(i \geq 2)$ are renormalization scheme dependent. We will now explicitly show how $R_{A}$ in Eq. (14) depends on the parameters $c_{i}$. Since $R_{A}$ arises from the sum over all perturbative terms, it should be independent of the choice of renormalization scheme. Therefore, one can write [17]

$$
\left(\frac{\partial}{\partial c_{i}}+\beta_{i}(a) \frac{\partial}{\partial a}\right) R_{A}=0
$$

Here $\beta_{i}(a)$ is defined as

$$
\frac{\partial a}{\partial c_{i}}=\beta_{i}(a)=-\hat{\beta}(a) \int_{0}^{a} \frac{x^{i+2}}{(\hat{\beta}(x))^{2}} d x
$$

which indicates how the coupling constant depends on the scheme parameters $c_{2}, c_{3}, \ldots$, where $\hat{\beta}(a)=\beta(a) / b$. The solution of this equation is [17]

$$
\frac{\partial a}{\partial c_{i}}=\frac{1}{i-1} a^{i+1}\left[1-\frac{(i-2)}{i} c a+\left(\frac{(i-1)(i-2)}{i(i+1)} c^{2}-\frac{(i-3)}{(i+1)} c_{2}\right) a^{2}+\cdots\right] .
$$

By setting $T_{n, 0} \equiv T_{n}$ in Eq. (10) and based on Eq. (24), one can derive the following result:

$$
\sum_{n=0}^{\infty} a^{n+1} \frac{\partial T_{n}}{\partial c_{i}}+(n+1) \beta_{i}(a) T_{n} a^{n}=0 .
$$

This leads to a set of nested equations for $T_{n}$ :

$$
\begin{aligned}
& \frac{\partial T_{0}}{\partial c_{i}}=0 \Rightarrow T_{0}=\tau_{0}=1 \\
& \frac{\partial T_{1}}{\partial c_{i}}=0 \Rightarrow T_{1}=\tau_{1}=\text { const }
\end{aligned}
$$

where we recall that $T_{0,0}=T_{0}=1$. 
For the $T_{2}$ coefficients we find

$$
\frac{\partial T_{2}}{\partial c_{2}}+1=0 \Rightarrow T_{2}=-c_{2}+\tau_{2},
$$

where $\tau_{2}$ is again constant and scheme invariant.

For the $T_{3}$ coefficient, we have the following set of two equations:

$$
\begin{gathered}
\frac{\partial T_{3}}{\partial c_{2}}+2 \tau_{1}=0, \\
\frac{\partial T_{3}}{\partial c_{3}}+\frac{1}{2}=0 .
\end{gathered}
$$

The simultaneous solution of these two differential equations is

$$
T_{3}=-2 c_{2} \tau_{1}-\frac{1}{2} c_{3}+\tau_{3}
$$

where again $\tau_{3}$ is a constant of integration and is scheme invariant.

For the $T_{4}$ coefficient, we get

$$
\begin{aligned}
\frac{\partial T_{4}}{\partial c_{2}}+\frac{1}{3} c_{2}+3 T_{2} & =0, \\
\frac{\partial T_{4}}{\partial c_{3}}+T_{1}-\frac{c}{6} & =0, \\
\frac{\partial T_{4}}{\partial c_{4}}+\frac{1}{3} & =0 .
\end{aligned}
$$

Simultaneously solving the differential equations in Eq. (32) gives the following result:

$$
T_{4}=-\frac{1}{3} c_{4}-c_{3}\left(\tau_{1}-\frac{c}{6}\right)+\frac{4}{3} c_{2}^{2}-3 c_{2} \tau_{2}+\tau_{4},
$$

where once again $\tau_{4}$ is a constant of integration and is RS invariant.

Following the procedure introduced in Ref. [10], we consider two specific choices of renormalization scheme. In the first scheme, the $c_{i}$ are selected so that $T_{n}=0(n \geq 2)$. With this choice, from Eqs. (28), (29), (31), and (33) we find that

$$
\begin{aligned}
& c_{2}=\tau_{2}, \\
& c_{3}=2\left(-2 c_{2} \tau_{1}+\tau_{3}\right), \\
& c_{4}=-\frac{3}{2} c_{3}\left(-\frac{c}{3}+2 \tau_{1}\right)+4 c_{2}^{2}-9 c_{2} \tau_{2}+3 \tau_{4} .
\end{aligned}
$$

In this case, the expansion series in Eq. (10) [or, equivalently, Eq. (23)] contains just two terms [10]:

$$
R_{(1)}=a_{(1)}+\tau_{1} a_{(1)}^{2}\left(\ln \frac{Q}{\Lambda}\right)
$$

where the coupling $a_{(1)}$, in addition to the universal parameters $b$ and $c$, depends also on $c_{2}, c_{3}$, and the other scheme parameters. It should be noted that since Eq. (23) is independent of the renormalization scale $\mu$, the finite expansion in Eq. (35) has been written in terms of the physical energy scale $Q$. As can be seen, the result for the observable $R$ is scheme independent since $\tau_{1}$ is RS invariant and the coupling constant $a_{(1)}$ is independent of the renormalization scale.

In the second scheme, the scheme parameters are selected so that $c_{i}=0(i \geq 2)$, which corresponds to the 't Hooft scheme [18]. With this choice, considering again Eqs. (28), (29), (31), and (33) will lead to $T_{n}=\tau_{n}$. In this case, Eq. (10) contains the infinite series

$$
\begin{aligned}
R_{(2)}= & \sum_{n=0}^{\infty} \tau_{n} a_{(2)}^{n+1}\left(\ln \frac{Q}{\Lambda}\right)=a_{(2)}\left(\ln \frac{Q}{\Lambda}\right)+\tau_{1} a_{(2)}^{2}\left(\ln \frac{Q}{\Lambda}\right) \\
& +\tau_{2} a_{(2)}^{3}\left(\ln \frac{Q}{\Lambda}\right)+\cdots
\end{aligned}
$$

Like the expansion in Eq. (35) and for similar reasons, only the physical energy scale $Q$ appears in the series expansion of Eq. (36), while $\tau_{1}, \tau_{2}$, etc., are RS invariants.

The coupling $a_{(2)}$ in Eq. (36) is obtained from the solution of the QCD $\beta$ function, where we only keep the two universal parameters $b$ and $c$. Therefore, it can be expressed in terms of the Lambert $W$ function. In particular, by using Eq. (5) the general solution reduces to

$$
\begin{aligned}
\frac{1}{a}+c \ln \left(\frac{c a}{1+c a}\right)= & \tau-\int\left(\frac{1}{-a^{2}\left(1+c a+c_{2} a^{2}+\cdots\right)}\right. \\
& \left.+\frac{1}{a^{2}(1+c a)}\right) d a
\end{aligned}
$$

where $\tau$ is the RS parameter, defined by $\tau=b \ln \left(\frac{Q}{\Lambda}\right)$. By setting $c_{n}=0$ in Eq. (37), we get

$$
\frac{1}{a_{0}}+c \ln \left(\frac{c a_{0}}{1+c a_{0}}\right)=b \ln \left(\frac{Q}{\Lambda_{R}}\right)
$$

To solve this equation, the $W(Q)$ function is defined such that

$$
1+W(Q)=-\frac{1}{c a_{0}} .
$$

Here $a_{0}=a_{0}(Q)$. Finally, one can write 


$$
\begin{gathered}
-\frac{1}{c a_{0}}-\ln \left(\frac{c a_{0}}{1+c a_{0}}\right)=\ln \left(\frac{Q}{\Lambda_{R}}\right)^{-b / c} \\
\Rightarrow W e^{W}=-\frac{1}{e}\left(\frac{Q}{\Lambda_{R}}\right)^{-b / c} .
\end{gathered}
$$

If $z(Q)=-\frac{1}{e}\left(\frac{Q}{\Lambda_{R}}\right)^{-b / c}$ is introduced, then Eq. (40) leads to

$$
W(z) e^{W(z)}=z
$$

The solution of this equation is called the Lambert $W$ function $[19,20]$. Then, from Eq. (39) we obtain

$$
a_{(2)} \equiv a_{0}\left(Q^{2}\right)=-\frac{1}{c\left[1+W_{-1}(z(Q))\right]} .
$$

Although two different cases for the McKeon et al. approach have been introduced, it can be shown that the expansions for $R_{(1)}$ and $R_{(2)}$ given by Eq. (35) and Eq. (36) are equivalent to each other. For this purpose, we consider two different couplings $a_{c}$ and $a_{d}$ which are evaluated using different renormalization schemes associated with the parameters $c_{i}$ and $d_{i}$, respectively [10],

$$
a_{c}=a_{d}+\lambda_{2}\left(c_{i}, d_{i}\right) a_{d}^{2}+\lambda_{3}\left(c_{i}, d_{i}\right) a_{d}^{3}+\cdots
$$

The couplings $a_{c}$ and $a_{d}$ are each satisfied by their related QCD $\beta$ functions such that

$$
\begin{aligned}
& \beta_{c}\left(a_{c}\right)=-b a_{c}^{2}\left(1+c a_{c}+c_{2} a_{c}^{2}+\cdots\right) \\
& \beta_{d}\left(a_{d}\right)=-b a_{d}^{2}\left(1+c a_{d}+d_{2} a_{d}^{2}+\cdots\right)
\end{aligned}
$$

Since $\frac{d a_{c}}{d d_{i}}=0$, this can be written as

$$
\left(\frac{\partial}{\partial d_{j}}+\beta_{j}\left(a_{d}\right) \frac{\partial}{\partial a_{d}}\right) \sum_{n=1}^{\infty} \lambda_{n}\left(c_{i}, d_{i}\right) a_{d}^{n}=0
$$

Using the boundary condition $\lambda_{n}\left(c_{i}, c_{i}\right)=0$, a set of differential equations can be obtained for $\lambda_{n}$ whose solutions lead to the following relation for the couplings:

$$
\begin{aligned}
a_{c}= & a_{d}-\left(d_{2}-c_{2}\right) a_{d}^{3}-\frac{1}{2}\left(d_{3}-c_{3}\right) a_{d}^{4} \\
& +\left[-\frac{1}{6}\left(d_{2}^{2}-c_{2}^{2}\right)+\frac{3}{2}\left(d_{2}-c_{2}\right)^{2}\right. \\
& \left.+\frac{c}{6}\left(d_{3}-c_{3}\right)-\frac{1}{3}\left(d_{4}-c_{4}\right)\right] a_{d}^{5}+\cdots
\end{aligned}
$$

Substituting this equation into Eq. (35) and using the 't Hooft scheme in which $d_{i}=0(i \geq 2)$, the expansion for $R_{(1)}$ reads

$$
\begin{aligned}
R_{(1)}= & a_{d}+c_{2} a_{d}^{3}+(1 / 2) c_{3} a_{d}^{4} \\
& +\tau_{1}\left(a_{d}+c_{2} a_{d}^{3}+(1 / 2) c_{3} a_{d}^{4}\right)^{2} .
\end{aligned}
$$

Evaluating $c_{i}$ from Eq. (34) in terms of the $\tau_{i}$ 's, substituting them into the above equation, and properly rearranging the desired series expansion, we obtain [10]

$$
R_{(1)}=a_{(2)}+\tau_{1} a_{(2)}^{2}+\tau_{2} a_{(2)}^{3}+\tau_{3} a_{(2)}^{4}+\cdots
$$

which is corresponding exactly to the series expansion for $R_{(2)}$ in Eq. (36). We recall that the coupling $a_{d}$ in Eq. (47) is evaluated for scheme parameters $d_{i}=0(i \geq 2)$. Therefore, $a_{(2)}$ is given in terms of the Lambert $W$ function, and this gives us full agreement between Eqs. (35) and (36).

\section{CONSIDERING OBSERVABLES IN THE MCKEON Et Al. AND CORGI APPROACHES}

After obtaining the required mathematical framework for the McKeon et al. approach, we now examine it by considering two QCD observables. We calculate them numerically and compare them with the results obtained from the CORGI approach. We first employ the McKeon et al. approach to obtain the ratio of cross sections for electron-positron annihilation into hadrons at a center-ofmass energy $\sqrt{s}=31.6 \mathrm{GeV}$.

\section{A. Electron-positron annihilation in the McKeon et al. approach}

The expansion up to fourth order of the ratio for electronpositron annihilation $R_{e^{+}} e^{-}$into hadrons with $N_{f}=5$ can be written as $[21,22]$

$$
\begin{aligned}
R_{e^{+} e^{-}}(s)= & \frac{11}{3}\left[1+a_{s}+1.40902 a_{s}^{2}-12.80 a_{s}^{3}\right. \\
& \left.-80.434 a_{s}^{4}+\cdots\right] .
\end{aligned}
$$

According to the notation of the McKeon et al. approach, one can write

$T_{0}=1, \quad T_{1}=1.409, \quad T_{2}=-12.80, \quad T_{3}=-80.434$

Using Eqs. (28), (29), and (31), the numerical values for the required $\mathrm{RS}$ invariants are given by

$\tau_{1}=T_{1}=1.409, \quad \tau_{2}=T_{2}+c_{2}=-11.3252$,
$\tau_{3}=T_{3}+\frac{1}{2} c_{3}+2 c_{2} \tau_{1}=-71.3600$.

We take the number of active quark flavors $N_{f}=5$ at a colliding energy $\sqrt{s}=31.6 \mathrm{GeV}$ and consider the QCD cutoff value in the $\overline{\mathrm{MS}}$ scheme as $\Lambda_{\overline{M S}}=419_{-168}^{+222} \mathrm{MeV}$, which is determined from the empirical value for $R_{e^{+}} e^{-}$[2]. 
The final numerical result for the ratio of the cross section in the McKeon et al. approach up to fourth order is

$$
\frac{3}{11} R_{e^{+} e^{-}}=1+a+\tau_{1} a^{2}+\tau_{2} a^{3}+\tau_{3} a^{4}=1.0556_{-0.006}^{+0.006},
$$

which is in good agreement with its available experimental value $R_{e^{+} e^{-}}=1.0527_{-0.005}^{+0.005}$ [23]. We recall that the coupling constant $a$ in Eq. (52) is given in terms of the Lambert $W$ function [see Eq. (42)].

There is a possibility to obtain a more precise numerical value for $R_{e^{+} e^{-}}$if we first do the calculations in Euclidean space, and then take the contour-improved result back to Minkowski space. This is because the perturbative coefficients for the concerned observable are computed more precisely in Euclidean space. In this regard, we need a relation between the ratio for $e^{+} e^{-}$annihilation and the Adler $D$ function in Euclidean space, which can be found in Ref. [24],

$$
D\left(Q^{2}\right)=Q^{2} \int_{4 m_{\pi}^{2}}^{\infty} \frac{R_{e^{+} e^{-}}(\mathcal{E})}{\left(\mathcal{E}+Q^{2}\right)^{2}} \mathrm{~d} \mathcal{E} .
$$

The Adler $D$ function has the following expansion:

$$
D\left(Q^{2}\right)=a\left(1+\sum_{n>0} d_{n} a^{n}\right),
$$

where the coefficients $d_{i}$ can be found in Refs. [21,22]. Nevertheless, if one is interesting, can do some computations which finally yields the $d_{i}$ coefficients in terms of the $r_{i}$ coefficients. Similar computations for the Higgs decay width to a gluon pair are shown in Sec. IV B. Doing the same calculations but for $R_{e^{+} e^{-}}$leads to

$$
\begin{aligned}
& d_{1}=r_{1}, \quad d_{2}=r_{2}+\frac{1}{3} \beta_{0}^{2} \pi^{2}, \\
& d_{3}=r_{3}+\pi^{2} r_{1} \beta_{0}^{2}+\frac{5}{6} \beta_{0} \pi^{2} \beta_{1} .
\end{aligned}
$$

From Eqs. (28), (29), and (31) one can obtain $\tau_{i}$ as follows, where in these equations the $T_{i}$ are replaced by $d_{i}$ :

$$
\tau_{1}=d_{1}, \quad \tau_{2}=d_{2}+c_{2}, \quad \tau_{3}=d_{3}+\frac{1}{2} c_{3}+2 c_{2} d_{1} .
$$

We take the result back to Minkowski space via analytic continuation, and then the observable $R$ can be written as [25]

$$
R(s)=\frac{1}{2 \pi} \int_{-\pi}^{\pi} W(\theta) D\left(s e^{i \theta}\right) d \theta .
$$

Here $W(\theta)$ is the weight function, which is taken to be 1 for $R_{e^{+} e^{-}}$. The contour-improved numerical result, taking
$\Lambda_{\overline{M S}}=419_{-168}^{+222} \mathrm{MeV}$ at the center-of-mass energy $\sqrt{s}=$ $31.6 \mathrm{GeV}$, is $\frac{3}{11} R_{e^{+} e^{-}}=1.0547_{-0.005}^{+0.005}$ which is, as expected, in better agreement with the available experimental data $\frac{3}{11} R_{e^{+} e^{-}}^{\exp }(\sqrt{s}=31.6 \mathrm{GeV})=1.0527_{-0.005}^{+0.005}[23]$.

If we take $\Lambda_{\bar{M} S}=210_{-14}^{+14} \mathrm{MeV}$, which corresponds to the world average value $\alpha_{s}\left(M_{Z}\right)=0.1181$ [26], then we get $\frac{3}{11} R_{e^{+} e^{-}}=1.0471_{-0.0007}^{+0.0006}$. The related conventional value for this observable is $\frac{3}{11} R_{e^{+} e^{-}}=1.0461_{-0.0008}^{+0.0015}$ [5].

Since there are experimental data for $R_{e^{+} e^{-}}$up to a center-of-mass energy of $208 \mathrm{GeV}$ [27] or even more, we also do the required calculations in the McKeon et al. approach, taking $\Lambda_{\overline{M S}}=210_{-14}^{+14} \mathrm{MeV}$ at the energy scales 42.5 and $56.5 \mathrm{GeV}$. The values we obtain for $\frac{3}{11} R_{e^{+} e^{-}}$at these energy scales are 1.0463 and 1.0441, respectively, which are comparable with their experimental values, i.e., 1.0554 [28] and 1.0745 [29]. A summery of the numerical results is given in Table I. It seems that as the energy scale increases the experimental data gradually diverges from the theoretical prediction, which was also seen in Ref. [30] (see their Fig. 8.4). But we should note that this conclusion is in fact based on only photon exchange. If a full theory is taken into account, including the electroweak contribution from $e^{+} e^{-}$annihilation into $Z$ boson, then it is expected to achieve good agrement with available experimental data.

\section{B. Electron-positron annihilation in the CORGI approach}

Here we do the same calculation for electron-positron annihilation into hadrons, but using the CORGI approach. The required information for this approach can be found in Refs. $[8,9,15]$. We recall that in this approach, using the self-consistency principle and taking the scheme parameters and renormalization scale into account, it is possible to obtain an expression for the perturbative coefficients of QCD observables at high order in terms of coefficients at lower orders. Then, by doing the resummation over the perturbative expansion but at a specified order, the resummed result at next-to-leading order (NLO), next-to-NLO (NNLO), etc., is RS invariant, and the renormalization scale is removed. Hence, there is a possibility to reconstruct the perturbative series in terms of RS invariants, and this new constructed series also does not depend on the renormalization scale as an unphysical parameter. Thus, we are now able to calculate the numerical result for the ratio $R_{e^{-} e^{+}}$using the CORGI approach.

The full theoretical expression for the electron-positron annihilation into hadrons can be written as [31]

$$
R(s)=N \sum_{f} Q_{f}^{2}\left(1+\frac{3}{4} C_{F} \tilde{R}(s)\right)+\left(\sum_{f} Q_{f}\right)^{2} \bar{R}(s),
$$

where $\tilde{R}$ is the perturbative corrections to the parton model result and has the following expansion: 
TABLE I. Numerical results for $R_{e^{+} e^{-}}$at $\sqrt{s}=31.6,42.5$, and $56.5 \mathrm{GeV}$, using the two different approaches.

\begin{tabular}{lccrcr}
\hline \hline & McKeon et al. approach & CORGI approach & Conventional pQCD & Experimental value & $\Lambda_{\overline{M S}}$ \\
\hline$R_{e^{+} e^{-}}(\sqrt{s}=31.6 \mathrm{GeV})$ & $1.04711_{-0.0007}^{+0.0006}$ & $1.04615_{-0.00005}^{+0.00003}$ & $1.04617_{-0.00008}^{+0.00015}$ & $1.0527_{-0.005}^{+0.005}$ & $210 \pm 14 \mathrm{MeV}$ \\
$R_{e^{+} e^{-}}(\sqrt{s}=42.5 \mathrm{GeV})$ & $1.0463_{-0.0004}^{+0.0004}$ & $1.0436_{-0.0005}^{+0.0004}$ & $1.0437_{-0.0005}^{+0.0004}$ & $1.0554 \pm 0.2$ & $210 \pm 14 \mathrm{MeV}$ \\
$R_{e^{+} e^{-}}(\sqrt{s}=52.5 \mathrm{GeV})$ & $1.0441_{-0.0004}^{+0.0005}$ & $1.0425_{-0.0004}^{+0.00045}$ & $1.0424_{-0.0004}^{+0.0004}$ & $1.0745 \pm 0.11$ & $210 \pm 14 \mathrm{MeV}$ \\
\hline \hline
\end{tabular}

$$
\tilde{R}(s)=a\left(1+\sum_{n} r_{n} a^{n}\right) .
$$

In Eq. (58) $\bar{R}$ refers to the "light-by-light" contribution and, because the factor $\sum_{f} Q_{f}^{2}$ is zero for light-quark flavors, it would is eliminated from the calculations.

In Minkowski space, using the CORGI approach the perturbative part of observable can be written as [9]

$$
\tilde{R}(s)=a_{0}+X_{2} a_{0}^{3}+X_{3} a_{0}^{4}+\cdots
$$

$X_{2}$ and $X_{3}$ are scheme-invariant quantities and, according to the notation of the McKeon et al. approach, can be written in terms of the coefficients $T_{i}$ [see Eq. (12)]. The explicit expressions for these quantities were given in Ref. [32] [see their Eq. (26)]. Then, we obtain our numerical results:

$$
X_{2}=-15.0870, \quad X_{3}=-16.6423 .
$$

By substituting the above numerical values into Eq. (60) (where $a_{0}$ is the coupling constant at the two-loop level written in terms of Lambert $W$ function) and then inserting Eq. (60) into Eq. (58), we determine that the value of $R_{e^{-} e^{+}}$in Minkowski space is $R_{e^{+} e^{-}}(\sqrt{s}=31.6 \mathrm{GeV})=$ $1.05440 \pm 0.006$. As before, we have set $\Lambda_{\overline{M S}}=$ $419_{-168}^{+222} \mathrm{MeV}$ and the energy scale $\sqrt{s}=31.6 \mathrm{GeV}$.

If one decides to do first the calculations in Euclidean space, the connection between the Adler $D$ function in this space and the observable $\tilde{R}(s)$ in Minkowski space [which is like Eq. (53)] is again needed. The perturbative expansion of the Adler $D$ function in Eq. (54) has the following form in the CORGI approach:

$$
\tilde{D}(s)=a_{0}+X_{2} a_{0}^{3}+X_{3} a_{0}^{4}+\cdots+X_{n} a_{0}^{n+1} .
$$

Here, the $X_{i}$ 's are RS invariants and can be written in term of $d_{i}$ [9]. The numerical values $X_{2}$ and $X_{3}$ for the Adler $D$ function are

$$
X_{2}=-7.2775, \quad X_{3}=39.9935 .
$$

Using the analytic continuation given by Eq. (57) and substituting Eq. (62) into it, the numerical value for the full expression $R(s)$ in Minkowski space can be obtained. The numerical result for the observable at NNLO using the
CORGI approach is $R_{e^{+} e^{-}}(\sqrt{s}=31.6 \mathrm{GeV})=1.0523 \pm$ 0.005 , where again $\Lambda_{\overline{M S}}=419_{-168}^{+222} \mathrm{MeV}$. This result is in better agreement with the reported experimental data $(1.0527 \pm 0.005$ [23]) than the result obtained from direct calculations in Minkowski space.

As in the previous approach, we calculate $\frac{3}{11} R_{e^{+} e^{-}}$in the CORGI approach at the energy scales 42.5 and $56.5 \mathrm{GeV}$, taking $\Lambda_{\overline{M S}}=210 \pm 14$. We obtain 1.0436 and 1.0425, respectively, while their experimental values are 1.0554 [28] and 1.0745 [29]. Similar calculations in the CORGI approach at $\sqrt{s}=31.6 \mathrm{GeV}$ but with $\Lambda_{\overline{M S}}=210 \pm 14$ have also been done. The related numerical values are listed in Table I.

\section{HIGGS DECAY GLOUN PAIR IN THE MCKEON Et Al. APPROACH}

As a second observable in the McKeon et al. approach we take into account the Higgs decay width to a gluon pair $(H \rightarrow g g)$. This observable is of fundamental phenomenological importance and its dominant contribution is given by [33]

$$
\Gamma(H \rightarrow g g)=\frac{4 G_{F} M_{H}^{3}}{9 \sqrt{2} \pi} R\left(M_{H}\right),
$$

where $G_{F}$ is the Fermi constant and $M_{H}$ is the Higgs boson mass. The numerical and perturbative series expansion of $R\left(M_{H}\right)$ is given later on in Secs. IV B and V, respectively.

As before, we first review how the scale renormalization is removed when we do a resummation over all perturbative terms of this observable. The RG summation procedure for $H \rightarrow g g$ is very similar to that for $R_{e^{+} e^{-}}$. Nonetheless, it is worth doing it again for this observable. According to the notation of the McKeon et al. approach, the expansion series of $R\left(M_{H}\right)$ has the following representation:

$$
R\left(M_{H}\right)=a_{s}^{2} \sum_{n=1}^{\infty} \sum_{m=0}^{n} T_{n, m} a_{s}^{n} L^{m},
$$

where $T_{0,0}=1$ and $L=\ln \frac{\mu}{M_{H}}$. Equation (65) depends on the renormalization scale $\mu$ as an unphysical quantity and, similarly to the perturbative expansion in Eq. (10), it is possible to show that all of the dependence on $\mu$ in $\Gamma(H \rightarrow g g)$ can be removed by summing over all 
logarithmic terms. We should note that the series expansion in Eq. (65) has an extra factor $a_{s}^{2}$ with respect to Eq. (10). In this regard, a different grouping is introduced:

$$
A_{n}(a)=\sum_{m=0}^{\infty} T_{n+m, n} a^{n+m+2}(n=0,1,2, \ldots) .
$$

Now Eq. (64) can be represented by

$$
\Gamma(H \rightarrow g g)=\frac{4 G_{F} M_{H}^{3}}{9 \sqrt{2} \pi} \sum_{n=0}^{\infty} A_{n}(a) L^{n}
$$

The RG equation implies that

$$
\left(\mu \frac{\partial}{\partial \mu}+\beta(a) \frac{\partial}{\partial a}\right) \Gamma(H \rightarrow g g)=0,
$$

which like before leads to

$$
A_{n}(a)=-\frac{\beta(a)}{n} \frac{d}{d a} A_{n-1}(a) .
$$

By introducing the $\eta$ scale, it follows that [11]

$$
\eta=\int_{a_{I}}^{a(\eta)} \frac{d x}{\beta(x)}, \quad a_{I}=\text { const. }
$$

Then,

$$
\beta(a) \frac{d}{d a}=\frac{d}{d \eta}
$$

By substituting Eq. (71) into Eq. (69), we obtain

$$
A_{n}(a)=-\frac{1}{n} \frac{d}{d \eta} A_{n-1}(a(\eta))
$$

where $\Lambda$ is defined by Eq. (18). Now one can show that $A_{0}, A_{1}, \ldots, A_{n}$ can be represented in terms of $A_{0}$. Therefore, we can write

$$
\begin{aligned}
A_{1} & =-1 \frac{d}{d \ln \frac{\mu}{\Lambda}} A_{0}, \\
A_{2} & =\frac{-1}{2} \frac{d}{d \ln \frac{\mu}{\Lambda}} A_{1}=\frac{-1}{2} \frac{d}{d \ln \frac{\mu}{\Lambda}}\left(\frac{-1}{1} \frac{d}{d \ln \frac{\mu}{\Lambda}} A_{0}\right) \\
& =\frac{1}{2} \frac{d^{2}}{d \ln ^{2} \frac{\mu}{\Lambda}} A_{0}, \\
A_{3} & =-\frac{1}{3} \frac{d}{d \ln \frac{\mu}{\Lambda}} A_{2}=-\frac{1}{3} \frac{d}{d \ln \frac{\mu}{\Lambda}} \frac{1}{1.2} \frac{d^{2}}{d \ln ^{2} \frac{\mu}{\Lambda}} A_{0} \\
& =\frac{-1}{1.2 .3} \frac{d^{3}}{d \ln ^{3} \frac{\mu}{\Lambda}} A_{0},
\end{aligned}
$$

$$
A_{n}=\frac{(-1)^{n}}{n !} \frac{d^{n}}{d^{n} \ln \frac{\mu}{\Lambda}} A_{0}
$$

If one defines $\eta=\ln \frac{\mu}{\Lambda}$, then

$$
A_{n}(a)=\frac{1}{n !}\left(-\frac{d}{d \eta}\right)^{n} A_{0}(a)
$$

and finally

$$
\begin{aligned}
R\left(M_{H}\right) & =\sum_{n=0}^{\infty} \frac{1}{n !}(-L)^{n} \frac{d^{n}}{d \eta^{n}} A_{0}(a(\eta)) \\
& =\exp \left(-L \frac{d}{d \eta}\right) A_{0}(a(\eta))
\end{aligned}
$$

The series expansion of the exponential term in Eq. (75) makes the right-hand side of this equation similar to the Taylor expansion of $A_{0}\left(a\left(\ln \frac{\mu}{\Lambda}-L\right)\right)$. For this purpose, as before one can write

$$
\begin{aligned}
A_{0}\left(a\left(\ln \frac{\mu}{\Lambda}-L\right)\right)= & A_{0}\left(a\left(\ln \frac{\mu}{\Lambda}\right)\right)-L A_{0}^{\prime}\left(a\left(\ln \frac{\mu}{\Lambda}\right)\right) \\
& +\frac{L^{2}}{2 !} A^{\prime \prime}{ }_{0}\left(a\left(\ln \frac{\mu}{\Lambda}\right)\right)+\cdots
\end{aligned}
$$

Consequently, the sum in Eq. (75) gives

$$
\begin{aligned}
R\left(M_{H}\right) & =A_{0}(a(\eta-L))=A_{0}\left(a\left(\ln \frac{\mu}{\Lambda}-\ln \frac{\mu}{M_{H}}\right)\right) \\
& =A_{0}\left(a\left(\frac{M_{H}}{\Lambda}\right)\right) .
\end{aligned}
$$

As shown by Eq. (77), the renormalization scale $\mu$ for the Higgs decay width to a gluon pair has been removed by summing over all of its perturbative series terms. 
Finally, using Eq. (66) with $m=0$, Eq. (77) becomes

$$
\Gamma(H \rightarrow g g)=\frac{4 G_{F} M_{H}^{3}}{9 \sqrt{2} \pi} a\left(\frac{M_{H}}{\Lambda}\right)^{2}\left(1+\sum_{n=1}^{\infty} T_{n} a\left(\frac{M_{H}}{\Lambda}\right)^{n}\right) .
$$

\section{A. Renormalization scheme dependence for $\boldsymbol{H} \rightarrow \boldsymbol{g g}$}

Here we specifically investigate how the series expansion for the Higgs decay width to a gluon pair can become RS invariant.

As before, the RS dependence of the expansion coefficients $T_{n}$ in Eq. (78) can be found by using the RG equation

$$
\left(\frac{\partial}{\partial c_{i}}+\beta_{i}(a) \frac{\partial}{\partial a}\right) \Gamma(H \rightarrow g g)=0,
$$

where $\beta_{i}(a)$ is given by Eq. (25). Considering the perturbative part of Higgs decay in Eq. (78) as

$$
R\left(M_{H}\right)=a^{2}+\sum_{n=1}^{\infty} T_{n} a^{n+2}
$$

and employing Eq. (79), we arrive at

$$
2 a \beta_{i}(a)+\sum_{n=1}^{\infty} a^{n+2} \frac{\partial T_{n}}{\partial c_{i}}+(n+2) a^{n+1} T_{n}=0
$$

This equation leads to a sequence of equations for $T_{n}$ order by order in terms of $a$, whose solutions are

$$
\begin{gathered}
\frac{\partial T_{1}}{\partial c_{i}}=0 \Rightarrow T_{1}=\lambda_{1}, \\
\frac{\partial T_{2}}{\partial c_{2}}+2=0 \Rightarrow T_{2}=-2 c_{2}+\lambda_{2},
\end{gathered}
$$

where $\lambda_{1}$ and $\lambda_{2}$ are constants of integration and RS invariants. For the coefficient $T_{3}$, one can write

$$
\begin{aligned}
\frac{\partial T_{3}}{\partial c_{2}}+3 T_{1} & =0 \\
\frac{\partial T_{3}}{\partial c_{3}}+1 & =0
\end{aligned}
$$

which leads to

$$
T_{3}=-3 \lambda_{1} c_{2}-c_{3}+\lambda_{3} .
$$

To obtain the $T_{4}$ coefficient, we need to derive the following differential equations

$$
\begin{aligned}
\frac{\partial T_{4}}{\partial c_{2}}+\frac{2 c_{2}}{3}+4 T_{2} & =0, \\
\frac{\partial T_{4}}{\partial c_{3}}-\frac{c}{3}+\frac{3}{2} T_{1} & =0, \\
\frac{\partial T_{4}}{\partial c_{4}}+\frac{2}{3} & =0,
\end{aligned}
$$

which leads to the final result

$$
T_{4}=\frac{11}{3} c_{2}^{2}-4 \lambda_{2} c_{2}+\frac{c}{3} c_{3}-\frac{3}{2} \lambda_{1} c_{3}-\frac{2}{3} c_{4}+\lambda_{4} .
$$

This process can be followed to obtain the other expressions for higher-order coefficients $T_{i}$.

As for $T_{1}$ and $T_{2}, \lambda_{3}$ and $\lambda_{4}$ in the coefficients of $T_{3}$ and $T_{4}$ are constants of integration and RS invariant, and they can be determined once $T_{i}$ and $c_{i}$ have been evaluated in some mass-independent RS.

Since the summation of the concerned perturbative series is scheme invariant, again two particular RSs are of special interest. In the first scheme one can set $c_{i}=0(i \geq 2)$, and consequently $T_{n}=\lambda_{n}$. In this case, Eq. (80) becomes

$$
R_{(2)}\left(M_{H}\right)=a_{(2)}^{2}+\sum_{n=1}^{\infty} \lambda_{n} a_{(2)}^{n+2} .
$$

In the second scheme, by setting $T_{i}=0(i \geq 2)$, Eq. (80) involves just two terms,

$$
R_{(1)}\left(M_{H}\right)=a^{2}+\lambda_{1} a^{3} .
$$

We recall that the coupling $a$ in Eq. (88) depends on the scheme parameters $c_{i}$, while the coupling Eq. (87) only depends on the two universal scheme parameters $b$ and $c$, which can be expressed in terms of the Lambert function $W(x)$, i.e., $x=W(x) e^{W(x)}$.

Again, considering the relation between the couplings in two different schemes, one can show that Eqs. (88) and (87) are equivalent, such that

$$
R_{(1)}\left(M_{H}\right)=R_{(2)}\left(M_{H}\right) .
$$

\section{B. Numerical value for the Higgs decay width to a gluon pair}

In this section we perform a numerical investigation of the Higgs decay width to a gluon pair in the McKeon et al. approach. The Higgs boson decay to a gluon pair is given by Eq. (64), where $R\left(M_{H}\right)$ in this equation has a numerical expansion in conventional perturbative QCD as follows [33]:

$$
\begin{aligned}
R\left(M_{H}\right)= & a^{2}\left(1+17.9167 a+153.15787 a^{2}\right. \\
& \left.+393.822 a^{3}+\cdots\right)
\end{aligned}
$$


TABLE II. Numerical results for Higgs decay to a gluon pair in the two different approaches.

\begin{tabular}{lccccc}
\hline \hline & $\begin{array}{c}\text { McKeon } \text { et al. } \\
\text { approach }\end{array}$ & $\begin{array}{c}\text { CORGI } \\
\text { approach }\end{array}$ & $\begin{array}{c}\text { Conventional } \\
\text { pQCD }\end{array}$ & $\begin{array}{c}\text { Experimental } \\
\text { value }\end{array}$ & $\Lambda_{\overline{M S}}$ \\
\hline$H \rightarrow g g\left(M_{H}=126 \pm 4\right)$ & $0.370_{-0.01}^{+0.01}$ & $0.379_{-0.02}^{+0.02}$ & $0.349_{-0.007}^{+0.007}$ & $\ldots$ & $210 \pm 14 \mathrm{MeV}$ \\
$H \rightarrow g g\left(M_{H}=125.18 \pm 0.16\right)$ & $0.369_{-0.020}^{+0.0018}$ & $0.378_{-0.0015}^{+0.0018}$ & $0.349_{-0.007}^{+0.007}$ & $\ldots$ & $210 \pm 14 \mathrm{MeV}$ \\
\hline \hline
\end{tabular}

According to the notation of the McKeon et al. approach, from Eq. (90) one can obtain the following numerical values for the coefficients $T_{i}$ :

$T_{1}=17.9167, \quad T_{2}=153.1578788, \quad T_{3}=393.822046$.

From Eqs. (82) and (84) we obtain the following numerical values for the $\lambda_{i}$ as RS invariants:

$$
\begin{aligned}
& \lambda_{1}=T_{1}=17.9167 \\
& \lambda_{2}=T_{2}+2 c_{2}=156.1074561 \\
& \lambda_{3}=T_{3}+c_{3}+3 c_{2} \lambda_{1}=482.927999 .
\end{aligned}
$$

Now, using the 't Hooft scheme, Eq. (87) becomes

$$
R\left(M_{H}\right)=a_{(2)}^{2}+\lambda_{1} a_{(2)}^{3}+\lambda_{2} a_{(2)}^{4}+\lambda_{3} a_{(2)}^{5}+\cdots
$$

In this equation, as illustrated before, the coupling constant $a_{(2)}$ can be written in terms of the Lambert $W$ function, which depends on the physical energy scale $Q$ and the $\lambda_{i}$ coefficients are scheme invariants. To numerically calculate the decay width $H \rightarrow g g$ in the McKeon et al. approach we consider again $G_{F}=1.16638 \times 10^{-5} \mathrm{GeV}^{-2}$ and the Higgs mass $M_{H}=126 \pm 4 \mathrm{GeV}$, while we take the number of active quark flavors $N_{f}=5$ and the QCD cutoff $\Lambda_{\overline{M S}}=$ $210 \pm 14 \mathrm{MeV}$. The value we obtain for the decay width is $\Gamma(H \rightarrow g g)=0.383 \pm 0.01 \mathrm{MeV}$, which can be compared to the conventional value $0.349 \pm 0.05 \mathrm{MeV}[34,35]$.

As for $R_{e^{+}, e^{-}}$, it is possible to perform the numerical calculation in Euclidian space, and then take the contourimproved result back to Minkowski space to get a more precise result.

The Adler $D$ function related to Eq. (93) has the following expansion:

$$
\begin{aligned}
D\left(M_{H}\right) & =a^{2}\left(1+\sum_{n=1}^{\infty} d_{n} a^{n}\right) \\
& =a^{2}+d_{1} a^{3}+d_{2} a^{4}+d_{3} a^{5}+\cdots
\end{aligned}
$$

We have the following relations between the coefficients $d_{i}$ and $T_{i}$ in Eq. (80), which we address in the next section:

$$
\begin{aligned}
& d_{1}=T_{1}, \quad d_{2}=T_{2}+\beta_{0}^{2} \pi^{2}, \\
& d_{3}=T_{3}+2 \pi^{2} T_{1} \beta_{0}^{2}+\frac{7}{3} \beta_{0} \pi^{2} \beta_{1} .
\end{aligned}
$$

Now, using Eqs. (82) and (84) we obtain the invariants $\lambda_{i}$ :

$\lambda_{1}=T_{1}, \quad \lambda_{2}=d_{2}+2 c_{2}, \quad \lambda_{3}=d_{3}+c_{3}+3 c_{2} \lambda_{1}$.

Finally, the perturbative part in Minkowski space converts to

$D\left(M_{H}\right)=a_{(2)}^{2}+\lambda_{1} a_{(2)}^{3}+\lambda_{2} a_{(2)}^{4}+\lambda_{3} a_{(2)}{ }^{5}+\cdots$,

where in addition to the RS invariants $\lambda_{i}$, the coupling $a_{2}$ (written in terms of the Lambert $W$ function) is independent of the renormalization scheme and depends only on the physical energy scale $Q=M_{H}$. Now the results for the Higgs decay width to a gluon pair in the McKeon et al. approach and in Euclidian space are accessible. We can get the decay width in Minkowski space by using the contourimproved integral:

$$
R\left(M_{H}\right)=\frac{1}{2 \pi} \int_{-\pi}^{\pi} D\left(M_{H} e^{i \theta}\right) d \theta .
$$

Finally, by taking $M(H)=126 \pm 4 \mathrm{GeV}$ and $\Lambda_{\mathrm{QCD}}^{n_{f}=5}=$ $210 \pm 14 \mathrm{MeV}$, the result is $\Gamma(H \rightarrow g g)=0.370 \pm$ $0.01 \mathrm{MeV}$, which is compatible with the conventional value $0.349 \pm 0.05 \mathrm{MeV}$. At the moment there is no experimental data for the Higgs decay width to a gluon pair, but we expect that the result determined from the contour-improved integral will be closer to the experimental value. Similar calculations with updated value for the Higgs mass, $M_{H}=125.18 \pm 0.16 \mathrm{GeV}$ [26] have also been done. The relevant numerical results are displayed in Table II.

\section{HIGGS DECAY TO A GLUON PAIR IN THE CORGI APPROACH}

Here we review how to obtain the result for Higgs decay to a gluon pair in the CORGI approach. More details can be found in Ref. [9]. Nevertheless, for the sake of clarity, some main steps to arrive at the CORGI approach for $\Gamma(H \rightarrow g g)$ 
are given here. We recall that in conventional perturbative series the Higgs decay width to a gluon pair is given by

$$
\begin{aligned}
\Gamma(H \rightarrow g g)= & \frac{4 G_{F} M_{H}^{3}}{9 \sqrt{2} \pi}\left[a_{s}^{2}+r_{1} a_{s}^{3},\right. \\
& \left.+r_{2} a_{s}^{4}+r_{3} a_{s}^{5}+\mathcal{O}\left(a_{s}^{6}\right)\right],
\end{aligned}
$$

where $a=\frac{\alpha_{s}}{\pi}$ is a coupling constant and the coefficients $r_{i}$ can be found in Ref. [33]. As it is obvious that the perturbative part of Eq. (99) is given by

$$
R=a^{2}+r_{1} a^{3}+r_{2} a^{4}+r_{3} a^{5}+\cdots
$$

The self-consistency principle for this observable up to $n$th order gives [17]

$$
\frac{\partial R^{(n)}}{\partial(\mathrm{RS})}=\mathcal{O}\left(\alpha_{s}^{n+1}\right)
$$

where $n=1,2, \ldots, n$ and RS stands for the scale parameter $\tau=b \ln \frac{\mu}{\Lambda}$ as well as the scheme parameters, such as $c_{2}, c_{3}, c_{4}, \ldots[9,15]$. Considering the first two terms in Eq. (100), that is,

$$
R=a^{2}+r_{1} a^{3}
$$

and using the self-consistency principe with respect to the variable $\tau$, we obtain

$$
\frac{\partial r_{1}}{\partial \tau}=2 \Rightarrow r_{1}-2 \tau=\rho_{1}
$$

Here $\rho_{1}$ is RS invariant and independent of the unphysical scale parameter $\tau$. Adding the third term to Eq. (102)m which contains the coefficient $r_{2}$, we obtain

$$
R=a^{2}+r_{1} a^{3}+r_{2} a^{4} .
$$

Then, by taking derivatives of $R$ with respect to the related $\mathrm{RS}$ parameters, one arrives at

$$
\begin{aligned}
& \frac{\partial r_{2}}{\partial c_{2}}=-2, \\
& \frac{\partial r_{2}}{\partial r_{1}}=c+\frac{3}{2} r_{1},
\end{aligned}
$$

where in deriving the first differential equation in above Eq. (26) has been used. The simultaneous solution of the differential equations (105) leads to

$$
r_{2}=c r_{1}+\frac{3}{4} r_{1}^{2}-2 c_{2}+X_{2}
$$

Here $X_{2}$ is a constant of integration and is RS invariant. If the forth term of the series expansion in Eq. (100) is considered, such that,

$$
R(Q)=a^{2}+r_{1} a^{3}+r_{2} a^{4}+r_{3} a^{5},
$$

then the self-consistency principle implies that

$$
\begin{aligned}
& \frac{\partial r_{3}}{\partial c_{2}}=-3 r_{1}, \\
& \frac{\partial r_{3}}{\partial c_{3}}=-1, \\
& \frac{\partial r_{3}}{\partial r_{1}}=\frac{3}{2} c r_{1}+2 r_{2}+c_{2} .
\end{aligned}
$$

Considering the above differential equations, the result for $r_{3}\left(r_{1}, c_{2}, c_{3}\right)$ is

$$
r_{3}\left(r_{1}, c_{2}, c_{3}\right)=\frac{r_{1}^{3}}{2}+\frac{7}{4} c r_{1}^{2}-3 c_{2} r_{1}+2 X_{2} r_{1}-c_{3}+X_{3},
$$

where $X_{3}$ is a constant of integration and is RS invariant. Substituting Eqs. (106) and (109) into Eq. (100), we get

$$
\begin{aligned}
R(Q)= & a^{2}+r_{1} a^{3}+\left(c r_{1}+\frac{3}{4} r_{1}^{2}-2 c_{2}+X_{2}\right) a^{4} \\
& +\left(\frac{r_{1}^{3}}{2}+\frac{7}{4} c r_{1}^{2}-3 c_{2} r_{1}+2 X_{2} r_{1}-c_{3}+X_{3}\right) a^{5}+\cdots
\end{aligned}
$$

Resuming the individual NLO, NNLO, etc., contributions in the above equation and employing the 't Hooft scheme at any specified order such that $c_{2}=c_{3}=\ldots=c_{n}=0$ (for convenience we set $r_{1}=0$ ), we arrive at the following result [9]:

$$
R(Q)=a_{0}^{2}+X_{2} a_{0}^{4}+X_{3} a_{0}^{5}+\cdots
$$

In this equation $a_{0}$ and $X_{i}$ are scale and scheme invariant and $a_{0}$ can be expressed in terms of the Lambert $W$ function. Now we can determine the numerical result for the Higgs decay width to a gluon pair directly in Minkowski space. To do this, we first need to calculate the numerical values for $X_{2}$ and $X_{3}$ in Eq. (111), which can be done using Eqs. (106) and (109) where the coefficients $r_{i}$ were calculated in Ref. [25]. The numerical results for these RS-invariant quantities are

$$
X_{2}=-107.2392, \quad X_{3}=3269.9755 .
$$

By substituting the above results into Eq. (111) and taking $M(H)=126 \pm 4 \mathrm{GeV}$ and $\Lambda_{\mathrm{QCD}}^{n_{f}=5}=210 \pm 10 \mathrm{MeV}$, the numerical result for the Higgs decay width is $\Gamma(H \rightarrow g g)=0.393 \pm 0.025 \mathrm{MeV}$.

Since the calculation can be done in Euclidean space similarly as for $R_{e^{+} e^{-}}$, we have to convert the calculations 
from Minkowski to Euclidean space. To do this, we need a relation between the coupling constants at different scales:

$$
\begin{aligned}
a(Q)= & a(\mu)\left(1+h_{1} a(\mu)+h_{2} a(\mu)^{2}\right. \\
& \left.+h_{3} a(\mu)^{3}+h_{4} a(\mu)^{4}+\cdots\right) .
\end{aligned}
$$

The following relations exist for the coefficients $h_{i}$ [36]:

$$
\begin{aligned}
& h_{1}=-\beta_{0} L, \quad h_{2}=\beta_{0}^{2} L^{2}-\beta_{1} L, \\
& h_{3}=-\beta_{0}^{3} L^{3}+\frac{5}{2} \beta_{0} \beta_{1} L^{2}-\beta_{2} L, \\
& h_{4}=\beta_{0}^{4} L^{4}-\frac{13}{3} \beta_{0}^{2} \beta_{1} L^{3}+3\left(\frac{1}{2} \beta_{1}^{2}+\beta_{0} \beta_{2}\right) L^{2}-\beta_{3} L .
\end{aligned}
$$

Here $L=\ln \left(\frac{s}{\mu^{2}}\right)$, where $s$ is a new scale and the $\beta_{i}$ 's are the coefficients of the QCD $\beta$ function. Substituting Eq. (113) into Eq. (100) will yield the observable $R$ in terms of the variable $s$. Using the dispersion relation (53) but for the Higgs decay width to a gluon pair will yield this observable in Euclidian space. To move the result from the $\mu$ scale to the $Q$ scale, we again use the displacement relation (113) in a proper state. Finally, we arrive at

$$
\begin{aligned}
D(Q)= & a(Q)^{2}+r_{1} a(Q)^{3}+\left(r_{2}+\beta_{0}^{2} \pi^{2}\right) a(Q)^{4} \\
& +\left(\frac{7}{3} \beta_{0} \beta_{1} \pi^{2}+r_{3}+2 \beta_{0}^{2} \pi^{2} r_{1}\right) a(Q)^{5}+O(a Q)^{6} .
\end{aligned}
$$

By comparing Eqs. (100) and (115), we obtain the following relations between the coefficients $d_{i}$ and $r_{i}$ :

$$
\begin{aligned}
& d_{1}=r_{1}, \quad d_{2}=r_{2}+\beta_{0}^{2} \pi^{2}, \\
& d_{3}=r_{3}+2 \pi^{2} r_{1} \beta_{0}^{2}+\frac{7}{3} \beta_{0} \pi^{2} \beta_{1} .
\end{aligned}
$$

We can get the decay width in Minkowski space by using the contour-improved integral:

$$
\Gamma(H \rightarrow g g)=\frac{4 G_{F} M_{H}^{3}}{9 \sqrt{2} \pi} \frac{1}{2 \pi} \int_{0}^{2 \pi}\left[a_{0}^{2}+X_{2} a_{0}^{4}+X_{3} a_{0}^{5}+\cdots\right] d \theta,
$$

where

$$
X_{2}=472.8741, \quad X_{3}=10096.8174
$$

To compute the above numerical results, we again need Eqs. (106) and (109) while the coefficients $r_{i}$ are replaced by $d_{i}$, given by Eq. (116). As before, $a_{0}\left(s_{0} e^{i \theta}\right)$ is the coupling constant in the CORGI approach. To numerically calculate the decay width $H \rightarrow g g$ in the CORGI approach, we take as before $G_{F}=1.16638 \times 10^{-5} \mathrm{GeV}^{-2}$ and the Higgs mass $M_{H}=126 \pm 4 \mathrm{GeV}$, while we take $N_{f}=5$ and $\Lambda_{\bar{M} S}=210 \pm 14 \mathrm{MeV}$. The value we obtain is $\Gamma(H \rightarrow g g)=0.379 \pm 0.03 \mathrm{MeV}$, which can be compared with the conventional result $0.349 \pm 0.05 \mathrm{MeV}[34,35]$. The numerical results, considering the updated value for the Higgs mass $M_{H}=125.18 \pm 0.16 \mathrm{GeV}$ [26], are shown in Table II.

\section{CONCLUSION}

In this article, we first reviewed the principles of the McKeon et al. approach [10-14]. This approach involves two essential steps. By defining a new summed group denoted by $A(n)$, we attempted to find a pattern that gives a recurrence relation between the $n$th and $(n-1)$ th order of $A(n)$ by taking the RGE into account. Finally, the concerned quantity at $n$th order can be written in terms of its expression at first order. In deriving this pattern the QCD $\beta$ function plays an essential role. Then, by constructing the primary series of the QCD observable in terms of $A(n)$ and resuming over all perturbative terms, the renormalization scale as an unphysical quantity disappears and the final result for the QCD observable depends only on the physical energy scale $Q$ [see Eq. (23)].

In the second step of this approach, the RS dependence of perturbative series coefficients were investigated. Considering the differential equation for the coupling constant with respect to the scheme parameters $c_{i},(i \geq 2)$, based on Eq. (25) one can rewrite the perturbative series of the QCD observable in terms of scheme-invariant quantities, while the coupling constant depends only on the physical energy scale, written in terms of the Lambert $W$ function [see Eq. (36)]. We recall that in this approach there is a possibility to render the series expansion RS invariant and scale independent, such that the series contains just two terms. However, in this case the related coupling constant is scale independent, but it depends on the scheme parameters $c_{2}, c_{3}$, etc., but overall the series is independent of the renormalization scale and scheme [see Eq. (35)]. At the end of Sec. II B it was shown that these two series expansions are equivalent.

In the second approach, called the CORGI approach, using the self-consistency principle there is a possibility to express the coefficient $r_{i}$ at the $n$th order in terms of the coefficients at lower orders, which finally includes just the coefficient at first order, i.e., $r_{1}$. When using this principle, we require the derivative of the observable with respect to the scheme parameters $c_{i}(i \geq 2)$ as well as the derivative with respect to $r_{1}$, such that in the $\frac{\partial R^{(i)}}{\partial(\mathrm{RS})}$ derivative the RSs can be labeled by the parameters $\left(r_{1}, c_{2}, c_{3}, \ldots\right)$ [17]. Later on, the parameter $r_{1}$ can be cast in terms of $\tau=\ln \frac{\mu}{\Lambda}$ as a scale parameter [9]. Using the self-consistency principle, the simultaneous solution of the differential equations obtained for $r_{i}$ can be represented as a function of the 
parameters $\left(r_{1}, c_{2}, c_{3}, \ldots\right)$. Rewriting the initial perturbative series of the QCD observable (2) in terms of the relations between the coefficients $r_{i}$, it is possible to resum the perturbative series NLO, and then at NNLO, and so on. Then, the desired perturbative series can be rewritten in terms of scheme invariants and coupling constants which only depend on the physical energy scale.

From this point of views, the CORGI and McKeon et al. approaches have achieved the same goal. Both of them were able to reconstruct the conventional perturbative series in terms of the scheme invariants and coupling constant, which does not depend on renormalization scheme. But, which one is more useful? At first, one may assign the priority to the McKeon et al. approach. In this approach the renormalization scale parameter and scheme parameters are investigated separately (as we explained in the Introduction), while in the CORGI approach this separation, utilizing the self-consistency principle, does not occur.

We reexamined these two approaches and used them to numerically compute the ratio $R_{e^{+} e^{-}}$and the Higgs decay width to a gluon pair. The numerical results, based on contour-improved integrations, are listed in Tables I and II. Inspecting Table I indicates that the results of the McKeon et al. approach are in better agreement with the available data.
In addition to these two approaches, there is another approach called the principle of maximum conformality (PMC). We talked about this approach in the Introduction. Based on this approach there is a possibility to absorb the nonconformal parts of the perturbative series coefficients into the renormalized coupling constant, and finally to attain a perturbation series in terms of only the conformal parts, while the renormalization scales can be fixed at any specified order. It is possible to convert the McKeon et al. approach to the PMC, which was done in Ref. [13]. Some other studies related to the PMC approach can be found in Refs. [6,7].

Converting the CORGI approach to the PMC approach is a difficult task, and we hope to report on it in the future. The McKeon et al. approach can be considered at an infrared fixed point where the QCD $\beta$ function is taken to be zero [37-39]. This subject is also interesting from other points of view, such as the infrared safe mode of QCD [40] or the AdS/CFT correspondence [41], and we hope to address these subjects in future research.

\section{ACKNOWLEDGMENTS}

The authors acknowledge Yazd University for providing the facilities necessary to do this project. The authors are indebted to M. R. Khellat for his reading of the manuscript and constructive comments.
[1] M. Mojaza, S. J. Brodsky, and X. G. Wu, Phys. Rev. Lett. 110, 192001 (2013).

[2] S. J. Brodsky, M. Mojaza, and X. G. Wu, Phys. Rev. D 89, 014027 (2014).

[3] Y. Ma, S. Q. Wang, H. B. Fu, H. H. Ma, S. J. Brodsky, and M. Mojaza, Rep. Prog. Phys. 78, 126201 (2015).

[4] A. Deur, J. M. Shen, X. G. Wu, and S. J. Brodsky, Phys. Lett. B 773, 98 (2017).

[5] J. M. Shen, X. G. Wu, B. L. Du, and S. J. Brodsky, Phys. Rev. D 95, 094006 (2017).

[6] A. L. Kataev, J. High Energy Phys. 02 (2014) 092.

[7] A. L. Kataev and S. V. Mikhailov, Teor. Mat. Fiz. 170, 174 (2012) [Theor. Math. Phys., 170139 (2012)].

[8] C. J. Maxwell, Nucl. Phys. B, Proc. Suppl. 86, 74 (2000).

[9] M. Akrami and A. Mirjalili, Phys. Rev. D 99, 074023 (2019).

[10] D. G. C. McKeon, Phys. Rev. D 92, 045031 (2015).

[11] F. Chishtie, D. G. C. McKeon, and T. N. Sherry, Phys. Rev. D 94, 054031 (2016).

[12] F. A. Chishtie and D. G. C. McKeon, Phys. Rev. D 95, 116013 (2017).

[13] F. A. Chishtie and D. G. C. McKeon, arXiv:1610.06487.

[14] D. G. C. McKeon, Can. J. Phys. 96, 1205 (2018).

[15] C. J. Maxwell and A. Mirjalili, Nucl. Phys. B577, 209 (2000).
[16] J. B. Kogut, R. B. Pearson, and J. Shigemitsu, Phys. Rev. Lett. 43, 484 (1979).

[17] P. M. Stevenson, Phys. Rev. D 23, 2916 (1981).

[18] G.'t Hooft, in The Whys of Subnuclear Physics, edited by A. Zichichi (Plenum, New York, 1977).

[19] B. Magradze, Conf. Proc. C980518, 158 (1999); Int. J. Mod. Phys. A 15, 2715 (2000).

[20] E. Gardi, G. Grunberg, and M. Karliner, J. High Energy Phys. 07 (1998) 007.

[21] K. G. Chetyrkin, J. H. Kuhn, and A. Kwiatkowski, Phys. Rep. 277, 189 (1996).

[22] P. A. Baikov, K. G. Chetyrkin, J. H. Kuhn, and J. Rittinger, J. High Energy Phys. 07 (2012) 017.

[23] R. Marshall, Z. Phys. C 43, 595 (1989).

[24] S. L. Adler, Phys. Rev. D 10, 3714 (1974).

[25] C. J. Maxwell and D. G. Tonge, Nucl. Phys. B535, 19 (1998).

[26] M. Tanabashi et al. (Particle Data Group), Phys. Rev. D 98, 030001 (2018).

[27] P. Achard et al. (L3 Collaboration) Eur. Phys. J. C 47, 1 (2006).

[28] B. Adeva et al. (Mark-J Collaboration) Phys. Rev. D 34, 681 (1986).

[29] K. Abe et al. (VENUS Collaboration) Phys. Lett. B 234, 382 (1990).

[30] D. Griffith, Introduction to Elementary Particles, 2nd ed. (Wiley-Vch, New York, 2008). 
[31] C. L. Basham, L. S. Brown, S. D. Ellis, and S. T. Love, Phys. Rev. D 17, 2298 (1978).

[32] A. Mirjalili and M. Akrami, Int. J. Mod. Phys. A 26, 2047 (2011).

[33] P. A. Baikov, K. G. Chetyrkin, and J. H. Kuhn, Phys. Rev. Lett. 96, 012003 (2006).

[34] K. G. Chetyrkin, Phys. Lett. B 390, 309 (1997).

[35] C. Anastasiou, F. Herzog, and A. Lazopoulos, J. High Energy Phys. 03 (2012) 035.
[36] M. Beneke, Nucl. Phys. B405, 424 (1993).

[37] T. Banks and A. Zaks, Nucl. Phys. B196, 189 (1982).

[38] J. Chyla, Phys. Rev. D 38, 3845 (1988).

[39] A. Hasenfratz, Phys. Rev. Lett. 108, 061601 (2012).

[40] L. Ghasemzadeh, A. Mirjalili, and S. Atshbar. Tehrani, Phys. Rev. D 100, 114017 (2019).

[41] S. J. Brodsky, G. F. de Teramond, and A. Deur, Phys. Rev. D 81, 096010 (2010). 